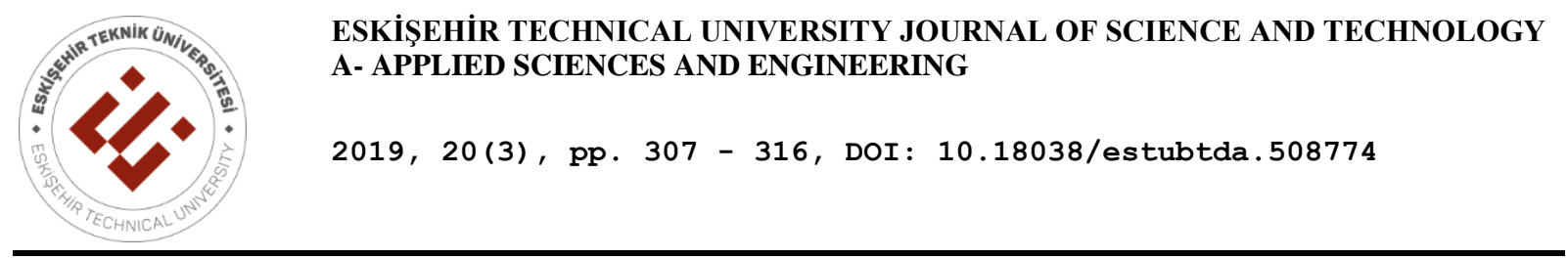

\title{
DIRECT PRODUCTS OF ROUGH SUBGROUPS
}

\section{Nurettin BAĞIRMAZ *}

\author{
Vocational School, Mardin Artuklu University, Mardin, Turkey
}

\begin{abstract}
In this research, the direct products of the rough approximations and rough subgroups in a group by direct product of normal subgroups are studied. In addition, some basic properties and homomorphic images of these structures are examined.
\end{abstract}

Keywords: Rough sets, Rough group, Rough subgroup, Direct product

\section{INTRODUCTION}

Z. Pawlak [1] first defined the theory of rough set as a significant mathematical instrument for forming and transaction the unfinished data in [1]. Since it was defined, the rough set theory evolved in various ways and was applied in different areas [2-4]. Some authors examined algebraic properties of rough sets, for example, Bonikowski [5], Pomykala and Pomykala [6] and Iwinski [7]. In [8], Kuroki and Wang studied the fundamental features of the rough approximations according to the normal subgroups. In recent years, many authors have studied the features of the rough approximations and subgroups according to the normal subgroups [9-14]. On the other hand, Biswas and Nanda [15] introduced a new definition of the notions of rough group. which were based only on the upper approximation. After this, Miao et al. [16] developed these concepts and proved their some new features. In addition, Bağırmaz and Özcan [17] gave new definition of rough semigroups groups on rough approximation spaces.

This study was configured in five sections. In section 2, the fundamental notions and results to be referred throughout the article were included. In section 3, the direct product of the rough approximations in a group was defined. We also gave some examples and examined their properties. In section 4, the direct product of the rough subgroups in a group was defined and some characteristics were given. Finally, in section 5 we examined the homomorphic images of the direct product of the rough subgroups.

\section{PRELIMINARIES}

In this section, we remember a few fundamental definitions and properties about rough approximations and direct product of groups to be used in this study. Throughout the study, G indicates a finite group with identity e.

Definition 1 [8] Let $N$ be a normal subgroup of $G$ and $A$ a non-empty subset of $G$. Let

$$
\bar{N}(A)=\{x \in G: x N \cap A \neq \emptyset\}, \quad \underline{N}(A)=\{x \in G: x N \subseteq A\} .
$$

Then $\bar{N}(A)$ and $\underline{N}(A)$ are called upper and lower approximations of $A$ with respect to the normal subgroup $N$, respectively.

*Corresponding Author:nurettinbagirmaz@artuklu.edu.tr

Received: 05.01.2019 Published: 26.09.2019 
Definition 2 [8] Let $N$ be a normal subgroup of a group $G$ and $A$ a non-empty subset of $G$. Then $A$ is called an upper rough subgroup (respectively, normal subgroup) of $G$ if $\bar{N}(A)$ is a subgroup (respectively, normal subgroup) of $G$. Similarly, $A$ is called a lower rough subgroup (respectively, normal subgroup) of $G$ if $\underline{N}(A)$ is a subgroup (respectively, normal subgroup) of $G$.

Theorem 3 [11] Let $N$ be a normal subgroup of a group $G$ and $A$ a non-empty subset of $G$. Then

$$
\bar{N}(A)=A N
$$

Theorem 4 [11] Let $N$ be a normal subgroup of a group $G$ and $A$ a subgroup of $G$. If $N \not \subset A$, then $\underline{N}(A)=\emptyset$; if $N \subseteq A$, then $\underline{N}(A)=A$.

Theorem 5 [11] Let $G_{1}$ and $G_{2}$ be two groups. Let $f$ be an epimorphism from $G_{1}$ to $G_{2}, N$ a normal subgroup of $G_{1}$ and $A$ a subgroup of $G_{1}$. Then

$$
\begin{aligned}
& \text { (1) } f(\underline{N}(A))=f(N)(f(A)), \\
& \text { (2) } f(\bar{N}(A))=\overline{(\overline{f(N)})(f(A)) .}
\end{aligned}
$$

Theorem 6 [11] Let $G_{1}$ and $G_{2}$ be two groups. Let $f$ be an epimorphism from $G_{1}$ to $G_{2}, N^{\prime}$ a normal subgroup of $G_{2}$ and $A^{\prime}$ a subgroup of $G_{2}$. Then

$$
\begin{aligned}
& \text { (1) } f^{-1}\left(\underline{N^{\prime}}\left(A^{\prime}\right)\right)=f^{-1}\left(N^{\prime}\right)\left(f^{-1}\left(A^{\prime}\right)\right), \\
& \text { (2) } f^{-1}\left(\overline{N^{\prime}}\left(A^{\prime}\right)\right)=\overline{\overline{f^{-1}\left(N^{\prime}\right)}}\left(f^{-1}\left(A^{\prime}\right)\right) .
\end{aligned}
$$

Theorem 7 [11] Let $G_{1}$ and $G_{2}$ be two groups. Let $f$ be an epimorphism from $G_{1}$ to $G_{2}, N$ and $A$ normal subgroups of $G_{1}$. Then

(1) If kerf $\subseteq N \subseteq A$, then $G_{1} / \underline{N}(A) \cong G_{2} / f(N)(f(A))$,

(2) If kerf $\subseteq N$, then $G_{1} / \bar{N}(A) \cong G_{2} / \overline{f(N)}(f(A))$.

Theorem 8 [19] Let $N_{1}$ and $N_{2}$ be normal subgroups of groups $G_{1}$ and $G_{2}$, respectively. Then $N_{1} \times N_{2}$ normal subgroups of groups $G_{1} \times G_{2}$ and $G_{1} \times G_{2} / N_{1} \times N_{2}=G_{1} / N_{1} \times G_{2} / N_{2}$.

\section{DIRECT PRODUCT OF ROUGH APPROXIMATIONS}

In this section, we will present direct product of rough approximations in a group according to the direct product of normal subgroups.

Definition 9 Let $N_{1}$ and $N_{2}$ be normal subgroups of groups $G_{1}$ and $G_{2}$, respectively. Let $A_{1}$ and $A_{2}$ be non-empty subsets of $G_{1}$ and $G_{2}$, respectively. Let

$$
\begin{gathered}
\overline{\left(N_{1} \times N_{2}\right)}\left(A_{1} \times A_{2}\right)=\left\{\left(g_{1}, g_{2}\right) \in G_{1} \times G_{2}:\left(g_{1}, g_{2}\right)\left(N_{1} \times N_{2}\right) \cap\left(A_{1} \times A_{2}\right) \neq \emptyset\right\}, \\
\underline{\left(N_{1} \times N_{2}\right)}\left(A_{1} \times A_{2}\right)=\left\{\left(g_{1}, g_{2}\right) \in G_{1} \times G_{2}:\left(g_{1}, g_{2}\right)\left(N_{1} \times N_{2}\right) \subseteq\left(A_{1} \times A_{2}\right)\right\} .
\end{gathered}
$$

Then $\overline{\left(N_{1} \times N_{2}\right)}\left(A_{1} \times A_{2}\right)$ and $\left(N_{1} \times N_{2}\right)\left(A_{1} \times A_{2}\right)$ are called upper and lower approximations of $A_{1} \times A_{2} \subseteq G_{1} \times G_{2}$ according to the normal subgroup $N_{1} \times N_{2}$ of $G_{1} \times G_{2}$, respectively. 
Let's explain this definition with the example below.

Example 10 Consider the groups $G_{1}=S_{3}=\{e,(12),(13),(23),(123),(132)\}$ and $G_{2}=\left(Z_{4}, \oplus\right)=$ $\{0,1,2,3\}$. Let $N_{1}=\{e,(123),(132)\}$ and $N_{2}=\{0,2\}$. Then $N_{1}$ and $N_{2}$ are normal subgroups of $G_{1}$ and $G_{2}$, respectively. A classification of $G_{1} \times G_{2}$ with respect to the normal subgroup $N_{1} \times N_{2}$ given below:

$$
\begin{gathered}
(e, 0)\left(N_{1} \times N_{2}\right)=\{(e, 0),(e, 2),((123), 0),((123), 2),((132), 0),((132), 2)\} \\
(e, 1)\left(N_{1} \times N_{2}\right)=\{(e, 1),(e, 3),((123), 1),((123), 3),((132), 1),((132), 3)\} \\
((12), 0)\left(N_{1} \times N_{2}\right)=\{((12), 0),((12), 2),((13), 0),((13), 2),((23), 0),((23), 2)\} \\
((12), 1)\left(N_{1} \times N_{2}\right)=\{((12), 1),((12), 3),((13), 1),((13), 3),((23), 1),((23), 3)\} .
\end{gathered}
$$

Let $A_{1}=\{e,(12),(123),(132)\}$ be a subset of $G_{1}$ and $A_{2}=\{0,2,3\}$ be a subset of $G_{2}$. Thus

and

$$
\underline{\left(N_{1} \times N_{2}\right)}\left(A_{1} \times A_{2}\right)=\{(e, 0),(e, 2),((123), 0),((123), 2),((132), 0),((132), 2)\}
$$

$$
\overline{\left(N_{1} \times N_{2}\right)}\left(A_{1} \times A_{2}\right)=G_{1} \times G_{2} .
$$

Using the definition of direct product of the rough approximations in a group, we can list several key features of direct product of the rough approximations in a group which are similar to properties of the rough approximations [8]. The proofs of these properties are all straightforward.

Proposition 11 Let $N_{1}$ and $N_{2}$ be normal subgroups of groups $G_{1}$ and $G_{2}$, respectively. Let $A_{1}$ and $B_{1}$ be non-empty subsets of $G_{1}$ and $A_{2}$ and $B_{2}$ be non-empty subsets of $G_{2}$. Let $A=A_{1} \times A_{2}$ and $B=$ $B_{1} \times B_{2}$. Then

$1\left(N_{1} \times N_{2}\right)(A) \subseteq A \subseteq \overline{\left(N_{1} \times N_{2}\right)}(A)$,

$2 \overline{\left(N_{1} \times N_{2}\right)}(A \cap B)=\underline{\left(N_{1} \times N_{2}\right)}(A) \cap \underline{\left(N_{1} \times N_{2}\right)}(B)$,

$3 \overline{\overline{\left(N_{1} \times N_{2}\right)}}(A \cup B)=\overline{\overline{\left(N_{1} \times N_{2}\right)}}(A) \cup \overline{\overline{\left(N_{1} \times N_{2}\right)}}(B)$,

$4 A \subseteq B \Rightarrow \underline{\left(N_{1} \times N_{2}\right)}(A) \subseteq \underline{\left(N_{1} \times N_{2}\right)}(B)$,

$\mathbf{5} A \subseteq B \Rightarrow \overline{\overline{\left(N_{1} \times N_{2}\right)}}(A) \subseteq \overline{\overline{\left(N_{1} \times N_{2}\right)}}(B)$,

$6\left(N_{1} \times N_{2}\right)(A \cup B) \supseteq \underline{\left(N_{1} \times N_{2}\right)}(A) \cup\left(N_{1} \times N_{2}\right)(B)$,

$7 \overline{\overline{\left(N_{1} \times N_{2}\right)}}(A \cap B) \subseteq \overline{\overline{\left(N_{1} \times N_{2}\right)}}(A) \cap \overline{\overline{\left(N_{1} \times N_{2}\right)}}(B)$.

Proposition 12 Let $N_{1}$ and $N_{2}$ be normal subgroups of groups $G_{1}$ and $G_{2}$, respectively. Let $A_{1}$ and $A_{2}$ be non-empty subsets of $G_{1}$ and $G_{2}$, respectively. Then

$$
\underline{\left(N_{1} \times N_{2}\right)}\left(A_{1} \times A_{2}\right)=\underline{N_{1}}\left(A_{1}\right) \times \underline{N_{2}}\left(A_{2}\right) .
$$

Proof. For any $\left(g_{1}, g_{2}\right) \in \underline{\left(N_{1} \times N_{2}\right)}\left(A_{1} \times A_{2}\right)$, we have

$$
\begin{aligned}
\underline{\left(N_{1} \times N_{2}\right)}\left(A_{1} \times A_{2}\right) & =\left\{\left(g_{1}, g_{2}\right) \in G_{1} \times G_{2}:\left(g_{1}, g_{2}\right)\left(N_{1} \times N_{2}\right) \subseteq A_{1} \times A_{2}\right\} \\
& =\left\{\left(g_{1}, g_{2}\right) \in G_{1} \times G_{2}:\left(g_{1} N_{1}\right) \times\left(g_{2} N_{2}\right) \subseteq A_{1} \times A_{2}\right\} \\
& =\left\{g_{1} \in G_{1}: g_{1} N_{1} \subseteq A_{1}\right\} \times\left\{g_{2} \in G_{2}: g_{2} N_{2} \subseteq A_{2}\right\} \\
& =\underline{N_{1}}\left(A_{1}\right) \times \underline{N_{2}}\left(A_{2}\right) .
\end{aligned}
$$

The proposition above shows that the lower approximation of the direct products of two sets is equal to the direct products of their lower approximations. 
Example 13 From Example 10, we obtain $\left(N_{1}\right)\left(A_{1}\right)=\{e,(123),(132)\}$ and $\left(N_{2}\right)\left(A_{2}\right)=\{0,2\}$. Then $\underline{\left(N_{1} \times N_{2}\right)}\left(A_{1} \times A_{2}\right)=\underline{\left(N_{1}\right)}\left(A_{1}\right) \times \underline{\left(N_{2}\right)}\left(\overline{\left.A_{2}\right)}\right.$.

Proposition 14 Let $N_{1}$ and $N_{2}$ be normal subgroups of groups $G_{1}$ and $G_{2}$, respectively. Let $A_{1}$ and $A_{2}$ be non-empty subsets of $G_{1}$ and $G_{2}$, respectively. Then

$$
\overline{\left(N_{1} \times N_{2}\right)(}\left(A_{1} \times A_{2}\right)=\overline{\left(N_{1}\right)}\left(A_{1}\right) \times \overline{\left(N_{2}\right)}\left(A_{2}\right) .
$$

Proof. For any $\left(g_{1}, g_{2}\right) \in \overline{\left(N_{1} \times N_{2}\right)}\left(A_{1} \times A_{2}\right)$, we have

$$
\begin{aligned}
\overline{\left(N_{1} \times N_{2}\right)\left(A_{1} \times A_{2}\right)} & =\left\{\left(g_{1}, g_{2}\right) \in G_{1} \times G_{2}:\left(g_{1}, g_{2}\right)\left(N_{1} \times N_{2}\right) \cap\left(A_{1} \times A_{2}\right) \neq \emptyset\right\} \\
& =\left\{\left(g_{1}, g_{2}\right) \in G_{1} \times G_{2}:\left(g_{1} N_{1} \times g_{2} N_{2}\right) \cap\left(A_{1} \times A_{2}\right) \neq \varnothing\right\} \\
& =\left\{\left(g_{1}, g_{2}\right) \in G_{1} \times G_{2}:\left(g_{1} N_{1} \cap A_{1}\right) \times\left(g_{2} N_{2} \cap A_{2}\right) \neq \varnothing\right\} \\
& =\left\{g_{1} \in G_{1}: g_{1} N_{1} \cap A_{1} \neq \emptyset\right\} \times\left\{g_{2} \in G_{2}: g_{2} N_{2} \cap A_{2} \neq \emptyset\right\} \\
& =\overline{\left(N_{1}\right)}\left(A_{1}\right) \times \overline{\left(N_{2}\right)}\left(A_{2}\right) .
\end{aligned}
$$

The above proposition shows that the upper approximation of the direct products of two sets is equal to the direct products of their upper approximations.

\section{DIRECT PRODUCT OF ROUGH SUBGROUPS}

Now, we will present the direct product of rough subgroups in a group according to the direct product of normal subgroups. For this purpose, we give some of their properties.

Definition 15 Let $N_{1}$ and $N_{2}$ be normal subgroups of groups $G_{1}$ and $G_{2}$, respectively. Let $A_{1}$ and $A_{2}$ be non-empty subsets of $G_{1}$ and $G_{2}$, respectively. If $\overline{\left(N_{1} \times N_{2}\right)}\left(A_{1} \times A_{2}\right)$ is a subgroup ( normal subgroup) of $G_{1} \times G_{2}$, then $A_{1} \times A_{2}$ is called a upper rough subgroup ( normal subgroup) of $G_{1} \times$ $G_{2}$. Similarly, if $\left(N_{1} \times N_{2}\right)\left(A_{1} \times A_{2}\right)$ is a subgroup (normal subgroup ) of $G_{1} \times G_{2}$, then $A_{1} \times A_{2}$ is called a lower rough subgroup (normal subgroup) of $G_{1} \times G_{2}$.

Proposition 16 Let $N_{1}$ and $N_{2}$ be normal subgroups of groups $G_{1}$ and $G_{2}$, respectively. Let $A_{1}$ and $A_{2}$ be subgroups of $G_{1}$ and $G_{2}$, respectively. If $N_{1} \times N_{2} \subseteq A_{1} \times A_{2}$, then $A_{1} \times A_{2}$ is a lower rough subgroup of $G_{1} \times G_{2}$.

Proof. Let $\left(e_{1}, e_{2}\right)$ be the identity of $G_{1} \times G_{2}$. Since $\left(e_{1}, e_{2}\right)\left(N_{1} \times N_{2}\right)=N_{1} \times N_{2} \subseteq A_{1} \times A_{2}$, then $\left(e_{1}, e_{2}\right) \in\left(N_{1} \times N_{2}\right)\left(A_{1} \times A_{2}\right)$. Let $\left(a_{1}, a_{2}\right)$ and $\left(b_{1}, b_{2}\right)$ be any elements of $\left(N_{1} \times N_{2}\right)\left(A_{1} \times A_{2}\right)$. Then $\left(a_{1}, a_{2}\right)\left(N_{1} \times N_{2}\right) \subseteq A_{1} \times A_{2}$ and $\left(b_{1}, b_{2}\right)\left(N_{1} \times N_{2}\right) \subseteq A_{1} \times A_{2}$. It is well known that $N_{1} \times N_{2}$ is a normal subgroup and $A_{1} \times A_{2}$ is a subgroup of $G_{1} \times G_{2}$. Then, we have

$$
\begin{aligned}
\left(a_{1} b_{1}, a_{2} b_{2}\right)\left(N_{1} \times N_{2}\right) & =\left(a_{1}, a_{2}\right)\left(b_{1}, b_{2}\right)\left(N_{1} \times N_{2}\right) \\
& =\left(\left(a_{1}, a_{2}\right)\left(N_{1} \times N_{2}\right)\left(b_{1}, b_{2}\right)\left(N_{1} \times N_{2}\right)\right) \subseteq\left(A_{1} \times A_{2}\right)\left(A_{1} \times A_{2}\right) \subseteq A_{1} \times A_{2} .
\end{aligned}
$$

This implies that

$$
\left(a_{1} b_{1}, a_{2} b_{2}\right) \in\left(N_{1} \times N_{2}\right)\left(A_{1} \times A_{2}\right) .
$$

Let $\left(a_{1}, a_{2}\right)$ be an arbitrary element of $\left(N_{1} \times N_{2}\right)\left(A_{1} \times A_{2}\right)$. Therefore

$$
\left.\left(a_{1}, a_{2}\right)=\left(a_{1} e_{1}, a_{2} e_{2}\right)=\overline{\left(a_{1}, a_{2}\right)\left(e_{1}\right.}, e_{2}\right) \in\left(a_{1}, a_{2}\right)\left(N_{1} \times N_{2}\right) \subseteq A_{1} \times A_{2}
$$

Since $A_{1} \times A_{2}$ is a subgroup of $G_{1} \times G_{2}$, we have $\left(a_{1}{ }^{-1}, a_{2}{ }^{-1}\right) \in A_{1} \times A_{2}$. 
Thus we have

$$
\left(a_{1}^{-1}, a_{2}^{-1}\right)\left(N_{1} \times N_{2}\right) \subseteq\left(A_{1} \times A_{2}\right)\left(A_{1} \times A_{2}\right) \subseteq A_{1} \times A_{2} .
$$

This implies that $\left(a_{1}^{-1}, a_{2}{ }^{-1}\right) \in\left(N_{1} \times N_{2}\right)\left(A_{1} \times A_{2}\right)$.

Hence $\left(N_{1} \times N_{2}\right)\left(A_{1} \times A_{2}\right)$ is a subgroup of $G_{1} \times G_{2}$.

Proposition 17 Let $N_{1}$ and $N_{2}$ be normal subgroups of groups $G_{1}$ and $G_{2}$, respectively. Let $A_{1}$ and $A_{2}$ be normal subgroups of $G_{1}$ and $G_{2}$, respectively. If $N_{1} \times N_{2} \subseteq A_{1} \times A_{2}$, then $A_{1} \times A_{2}$ is a lower normal subgroup of $G_{1} \times G_{2}$.

Proof. By Proposition 16, we showed that $A_{1} \times A_{2}$ was a lower rough subgroup $G_{1} \times G_{2}$. It is enough to show that $\left(N_{1} \times N_{2}\right)\left(A_{1} \times A_{2}\right)$ is normal subgroup of $G_{1} \times G_{2}$. Let $\left(a_{1}, a_{1}\right)$ and $\left(g_{1}, g_{2}\right)$ be arbitrary elemens of $\left(N_{1} \times N_{2}\right)\left(A_{1} \times A_{2}\right)$ and $G_{1} \times G_{2}$, respectively. Therefore, $\left(a_{1}, a_{2}\right)\left(N_{1} \times N_{2}\right) \subseteq$ $A_{1} \times A_{2}$. It is well known that $N_{1} \times N_{2}$ and $A_{1} \times A_{2}$ are normal subgroups of $G_{1} \times G_{2}$. Therefore, we have

$$
\begin{aligned}
\left(g_{1} a_{1} g_{1}^{-1}, g_{2} a_{2} g_{2}^{-1}\right)\left(N_{1} \times N_{2}\right) & =\left(g_{1}, g_{2}\right)\left(a_{1}, a_{2}\right)\left(g_{1}^{-1}, g_{2}^{-1}\right)\left(N_{1} \times N_{2}\right) \\
& =\left(g_{1}, g_{2}\right)\left(a_{1}, a_{2}\right)\left(g_{1}, g_{2}\right)^{-1}\left(N_{1} \times N_{2}\right) \\
& =\left(g_{1}, g_{2}\right)\left(\left(a_{1}, a_{2}\right)\left(N_{1} \times N_{2}\right)\right)\left(g_{1}, g_{2}\right)^{-1} \\
& \subseteq\left(g_{1}, g_{2}\right)\left(A_{1} \times A_{2}\right)\left(g_{1}, g_{2}\right)^{-1} \subseteq A_{1} \times A_{2}
\end{aligned}
$$

This implies that $\left(g_{1} a_{1} g_{1}^{-1}, g_{2} a_{2} g_{2}^{-1}\right) \in \underline{\left(N_{1} \times N_{2}\right)}\left(A_{1} \times A_{2}\right)$.

Hence $\left(N_{1} \times N_{2}\right)\left(A_{1} \times A_{2}\right)$ is a normal subgroup of $G_{1} \times G_{2}$.

Proposition 18 Let $N_{1}$ and $N_{2}$ be normal subgroups of groups $G_{1}$ and $G_{2}$, respectively. Let $A_{1}$ and $A_{2}$ be subgroups of $G_{1}$ and $G_{2}$, respectively. Then $A_{1} \times A_{2}$ is a upper rough subgroup of $G_{1} \times G_{2}$.

Proof. Let $\left(e_{1}, e_{2}\right)$ be the identity of $G_{1} \times G_{2}$. Since $N_{1} \times N_{2}$ and $A_{1} \times A_{2}$ are subgroups $G_{1} \times G_{2}$, we have $\left(e_{1}, e_{2}\right) \in A_{1} \times A_{2}$ and $\left(e_{1}, e_{2}\right) \in\left(e_{1}, e_{2}\right)\left(N_{1} \times N_{2}\right)$. Thus

$$
\left(e_{1}, e_{2}\right) \in\left(e_{1}, e_{2}\right)\left(N_{1} \times N_{2}\right) \cap A_{1} \times A_{2} .
$$

This implies that $\left(e_{1}, e_{2}\right) \in \overline{\left(N_{1} \times N_{2}\right)}\left(A_{1} \times A_{2}\right)$.

Let $\left(a_{1}, a_{2}\right)$ and $\left(b_{1}, b_{2}\right)$ be arbitrary elements of $\overline{\left(N_{1} \times N_{2}\right)}\left(A_{1} \times A_{2}\right)$. Therefore, there are the elements $\left(g_{1}, g_{2}\right)$ and $\left(h_{1}, h_{2}\right)$ in $G_{1} \times G_{2}$ such that

$$
\left(g_{1}, g_{2}\right) \in\left(a_{1}, a_{2}\right)\left(N_{1} \times N_{2}\right) \cap\left(A_{1} \times A_{2}\right)
$$

and

$$
\left(h_{1}, h_{2}\right) \in\left(b_{1}, b_{2}\right)\left(N_{1} \times N_{2}\right) \cap\left(A_{1} \times A_{2}\right) \text {. }
$$

Thus

Then

$$
\begin{gathered}
\left(g_{1}, g_{2}\right) \in\left(a_{1}, a_{2}\right)\left(N_{1} \times N_{2}\right),\left(h_{1}, h_{2}\right) \in\left(b_{1}, b_{2}\right)\left(N_{1} \times N_{2}\right), \\
\left(g_{1}, g_{2}\right) \in A_{1} \times A_{2} \text { and }\left(h_{1}, h_{2}\right) \in A_{1} \times A_{2} .
\end{gathered}
$$

$$
\left(g_{1}, g_{2}\right)\left(h_{1}, h_{2}\right)=\left(g_{1} h_{1}, g_{2} h_{2}\right) \in A_{1} \times A_{2}
$$

and 


$$
\left(g_{1}, g_{2}\right)\left(h_{1}, h_{2}\right) \in\left(\left(a_{1}, a_{2}\right)\left(N_{1} \times N_{2}\right)\right)\left(\left(b_{1}, b_{2}\right)\left(N_{1} \times N_{2}\right)\right) .
$$

Since $N_{1} \times N_{2}$ is a normal subgroup of $G_{1} \times G_{2}$ and $\left(g_{1}, g_{2}\right)\left(h_{1}, h_{2}\right)=\left(g_{1} h_{1}, g_{2} h_{2}\right)$, then

$$
\left(g_{1} h_{1}, g_{2} h_{2}\right) \in\left(\left(a_{1}, a_{2}\right)\left(b_{1}, b_{2}\right)\right)\left(N_{1} \times N_{2}\right)=\left(a_{1} b_{1}, a_{2} b_{2}\right)\left(N_{1} \times N_{2}\right) .
$$

Thus $\left(g_{1} h_{1}, g_{2} h_{2}\right) \in\left(a_{1} b_{1}, a_{2} b_{2}\right)\left(N_{1} \times N_{2}\right) \cap\left(A_{1} \times A_{2}\right)$. This implies that

$$
\left(a_{1} b_{1}, a_{2} b_{2}\right) \in \overline{\left(N_{1} \times N_{2}\right)}\left(A_{1} \times A_{2}\right) .
$$

Let $\left(a_{1}, a_{2}\right)$ be any element of $\overline{\left(N_{1} \times N_{2}\right)}\left(A_{1} \times A_{2}\right)$. Then

$$
\left(g_{1}, g_{2}\right) \in\left(a_{1}, a_{2}\right)\left(N_{1} \times N_{2}\right) \cap\left(A_{1} \times A_{2}\right)
$$

for some $\left(g_{1}, g_{2}\right) \in G_{1} \times G_{2}$, that is, $\left(g_{1}, g_{2}\right) \in\left(a_{1}, a_{2}\right)\left(N_{1} \times N_{2}\right),\left(g_{1}, g_{2}\right) \in A_{1} \times A_{2}$. Thus $\left(g_{1}, g_{2}\right)^{-1}=\left(g_{1}{ }^{-1}, g_{2}{ }^{-1}\right) \in A_{1} \times A_{2}$ and $\left(g_{1}, g_{2}\right)=\left(a_{1}, a_{2}\right)\left(n_{1}, n_{2}\right)$ for some $\left(n_{1}, n_{2}\right) \in N_{1} \times N_{2}$, and so $\left(n_{1}, n_{2}\right)^{-1}=\left(n_{1}{ }^{-1}, n_{2}{ }^{-1}\right) \in N_{1} \times N_{2}$. Since $N_{1} \times N_{2}$ is a normal subgroup of $G_{1} \times G_{2}$, we have

Thus

$$
\begin{aligned}
\left(g_{1}, g_{2}\right)^{-1} & =\left(\left(a_{1}, a_{2}\right)\left(n_{1}, n_{2}\right)\right)^{-1} \\
& =\left(n_{1}, n_{2}\right)^{-1}\left(a_{1}, a_{2}\right)^{-1} \in\left(N_{1} \times N_{2}\right)\left(a_{1}{ }^{-1}, a_{2}{ }^{-1}\right) \\
& =\left(a_{1}^{-1}, a_{2}{ }^{-1}\right)\left(N_{1} \times N_{2}\right) .
\end{aligned}
$$

and so

$$
\left(g_{1}, g_{2}\right)^{-1} \in\left(a_{1}, a_{2}\right)^{-1}\left(N_{1} \times N_{2}\right) \cap\left(A_{1} \times A_{2}\right),
$$

$$
\left(a_{1}, a_{2}\right)^{-1} \in \overline{\left(N_{1} \times N_{2}\right)}\left(A_{1} \times A_{2}\right) .
$$

Hence $\overline{\left(N_{1} \times N_{2}\right)}\left(A_{1} \times A_{2}\right)$ is a subgroup of $G_{1} \times G_{2}$.

Proposition 19 Let $N_{1}$ and $N_{2}$ be normal subgroups of groups $G_{1}$ and $G_{2}$, respectively. Let $A_{1}$ and $A_{2}$ be normal subgroups of $G_{1}$ and $G_{2}$, respectively. Then $A_{1} \times A_{2}$ is an upper rough normal subgroup of $G_{1} \times G_{2}$.

Proof. It is enough to show that $\overline{\left(N_{1} \times N_{2}\right)}\left(A_{1} \times A_{2}\right)$ is normal subgroup of $G_{1} \times G_{2}$. Let $\left(a_{1}, a_{2}\right)$ and $\left(g_{1}, g_{2}\right)$ be an arbitrary element of $\overline{\left(N_{1} \times N_{2}\right)}\left(A_{1} \times A_{2}\right)$ and $G_{1} \times G_{2}$, respectively. Therefore, there is an element $\left(h_{1}, h_{2}\right)$ in $G_{1} \times G_{2}$ such that $\left(h_{1}, h_{2}\right) \in\left(a_{1}, a_{2}\right)\left(N_{1} \times N_{2}\right) \cap\left(A_{1} \times A_{2}\right)$. Then $\left(h_{1}, h_{2}\right) \in\left(a_{1}, a_{2}\right)\left(N_{1} \times N_{2}\right)$ and $\left(h_{1}, h_{2}\right) \in\left(A_{1} \times A_{2}\right)$. Since $N_{1} \times N_{2}$ is normal,

$$
\begin{aligned}
\left(g_{1} h_{1} g_{1}{ }^{-1}, g_{2} h_{2} g_{2}{ }^{-1}\right) & =\left(g_{1}, g_{2}\right)\left(h_{1}, h_{2}\right)\left(g_{1}, g_{2}\right)^{-1} \in\left(g_{1}, g_{2}\right)\left(\left(a_{1}, a_{2}\right)\left(N_{1} \times N_{2}\right)\right)\left(g_{1}, g_{2}\right)^{-1} \\
& =\left(\left(g_{1}, g_{2}\right)\left(a_{1}, a_{2}\right)\right)\left(\left(N_{1} \times N_{2}\right)\left(g_{1}, g_{2}\right)^{-1}\right) \\
& =\left(\left(g_{1}, g_{2}\right)\left(a_{1}, a_{2}\right)\right)\left(\left(g_{1}, g_{2}\right)^{-1}\left(N_{1} \times N_{2}\right)\right) \\
& =\left(\left(g_{1}, g_{2}\right)\left(a_{1}, a_{2}\right)\left(g_{1}, g_{2}\right)^{-1}\right)\left(N_{1} \times N_{2}\right) \\
& =\left(g_{1} a_{1} g_{1}{ }^{-1}, g_{2} a_{2} g_{2}{ }^{-1}\right)\left(N_{1} \times N_{2}\right) .
\end{aligned}
$$

Since $\left(A_{1} \times A_{2}\right)$ is normal,

$$
\left(g_{1} h_{1} g_{1}{ }^{-1}, g_{2} h_{2} g_{2}{ }^{-1}\right)=\left(g_{1}, g_{2}\right)\left(h_{1}, h_{2}\right)\left(g_{1}, g_{2}\right)^{-1} \in\left(g_{1}, g_{2}\right)\left(A_{1} \times A_{2}\right)\left(g_{1}, g_{2}\right)^{-1} \subseteq A_{1} \times A_{2} .
$$

Thus

$$
\left(g_{1} h_{1} g_{1}^{-1}, g_{2} h_{2} g_{2}^{-1}\right) \in\left(g_{1} a_{1} g_{1}^{-1}, g_{2} a_{2} g_{2}^{-1}\right)\left(N_{1} \times N_{2}\right) \cap\left(A_{1} \times A_{2}\right),
$$


and so

$$
\left(g_{1} a_{1} g_{1}{ }^{-1}, g_{2} a_{2} g_{2}{ }^{-1}\right) \in \overline{\left(N_{1} \times N_{2}\right)}\left(A_{1} \times A_{2}\right)
$$

Hence $\overline{\left(N_{1} \times N_{2}\right)}\left(A_{1} \times A_{2}\right)$ is a normal subgroup of $G_{1} \times G_{2}$.

The following propositions give the characterization of the upper and lower approximations.

Proposition 20 Let $N_{1}$ and $N_{2}$ be normal subgroups of groups $G_{1}$ and $G_{2}$, respectively. Let $A_{1}$ and $A_{2}$ be normal subgroups of $G_{1}$ and $G_{2}$, respectively. If $N_{1} \times N_{2} \subseteq A_{1} \times A_{2}$, then

$$
\underline{\left(N_{1} \times N_{2}\right)}\left(A_{1} \times A_{2}\right)=A_{1} \times A_{2} .
$$

Proof. This can be easily got from Proposition 12 and Theorem 4.

Proposition 21 Let $N_{1}$ and $N_{2}$ be normal subgroups of groups $G_{1}$ and $G_{2}$, respectively. Let $A_{1}$ and $A_{2}$ be non-empty subsets of $G_{1}$ and $G_{2}$, respectively. Then

$$
\overline{\left(N_{1} \times N_{2}\right)}\left(A_{1} \times A_{2}\right)=A_{1} N_{1} \times A_{2} N_{2} .
$$

Proof. This can be easily got from Proposition 14 and Theorem 3.

\section{HOMOMORPHIC IMAGE OF DIRECT PRODUCTS OF ROUGH SUBGROUPS}

This last section is reserved into properties of the direct products of the rough approximations of a subgroup in a group under homomorphisms between the two groups.

Let $\varphi_{i}: G_{i} \rightarrow H_{i}$ be group homomorphisms ( $\left.\mathrm{i}=1,2\right)$. Define $\sigma$ by

$$
\begin{aligned}
\sigma: G_{1} \times G_{2} & \rightarrow H_{1} \times H_{2} \\
\left(g_{1}, g_{2}\right) & \mapsto\left(\varphi_{1}\left(g_{1}\right), \varphi_{2}\left(g_{2}\right)\right) .
\end{aligned}
$$

Then $\sigma$ is a homomorphism and $\operatorname{Ker} \sigma=\operatorname{Ker} \varphi_{1} \times \operatorname{Ker} \varphi_{2}, \operatorname{Im} \sigma=\operatorname{Im} \varphi_{1} \times \operatorname{Im} \varphi_{2}$. If $\varphi_{i}: G_{i} \rightarrow H_{i}$ is an isomorphism ( $\mathrm{i}=1,2)$, then $\sigma: G_{1} \times G_{2} \rightarrow H_{1} \times H_{2}$ is an isomorphism.

Proposition 22 Let $N_{1}$ and $N_{2}$ be normal subgroups of groups $G_{1}$ and $G_{2}$, respectively. Let $A_{1}$ and $A_{2}$ be normal subgroups of $G_{1}$ and $G_{2}$, respectively. Then

$$
\begin{aligned}
& (1) G_{1} \times G_{2} / \overline{\left(N_{1} \times N_{2}\right)}\left(A_{1} \times A_{2}\right) \cong G_{1} / \overline{N_{1}}\left(A_{1}\right) \times G_{2} / \overline{N_{2}}\left(A_{2}\right), \\
& (2) G_{1} \times G_{2} / \underline{\left(N_{1} \times N_{2}\right)}\left(A_{1} \times A_{2}\right) \cong G_{1} / \underline{N_{1}}\left(A_{1}\right) \times G_{2} / \underline{N_{2}}\left(A_{2}\right) .
\end{aligned}
$$

Proof. (1) By the Proposition 14,we get $\left.\overline{\left(N_{1} \times N_{2}\right)(} A_{1} \times A_{2}\right)=\overline{\left(N_{1}\right)}\left(A_{1}\right) \times \overline{\left(N_{2}\right)}\left(A_{2}\right)$. Since $A_{1}$ and $A_{2}$ are normal subgroups of $G_{1}$ and $G_{2}$, respectively, by Theorem 3 it follows that $\overline{\left(N_{1}\right)}\left(A_{1}\right)$ and $\overline{\left(N_{2}\right)}\left(A_{2}\right)$ are normal subgroups of $G_{1}$ and $G_{2}$, respectively. Then

$$
G_{1} \times G_{2} / \overline{\left(N_{1} \times N_{2}\right)}\left(A_{1} \times A_{2}\right)=G_{1} \times G_{2} /\left(\overline{\left(N_{1}\right)}\left(A_{1}\right) \times \overline{\left(N_{2}\right)}\left(A_{2}\right)\right) .
$$

By Theorem 8, we get

$$
G_{1} \times G_{2} / \overline{\left(N_{1} \times N_{2}\right)}\left(A_{1} \times A_{2}\right) \cong G_{1} / \overline{N_{1}}\left(A_{1}\right) \times G_{2} / \overline{N_{2}}\left(A_{2}\right),
$$


(2) By the Proposition 12, we get $\left(N_{1} \times N_{2}\right)\left(A_{1} \times A_{2}\right)=N_{1}\left(A_{1}\right) \times \underline{N_{2}}\left(A_{2}\right)$. Since $A_{1}$ and $A_{2}$ are normal subgroups of $G_{1}$ and $G_{2}$, respectively, by Theorem 4 it follows that $\underline{N}_{1}\left(A_{1}\right)$ and $\underline{N_{2}}\left(A_{2}\right)$ are normal subgroups of $G_{1}$ and $G_{2}$, respectively. Then

$G_{1} \times G_{2} / \underline{\left(N_{1} \times N_{2}\right)}\left(A_{1} \times A_{2}\right)=G_{1} \times G_{2} /\left(\underline{N_{1}}\left(A_{1}\right) \times \underline{N_{2}}\left(A_{2}\right)\right)$.

By the Theorem 8, we get

$$
G_{1} \times G_{2} / \underline{\left(N_{1} \times N_{2}\right)}\left(A_{1} \times A_{2}\right) \cong G_{1} / \underline{N_{1}}\left(A_{1}\right) \times G_{2} / \underline{N_{2}}\left(A_{2}\right) .
$$

Proposition 22 Let $\varphi_{i}: G_{i} \rightarrow H_{i}$ be group epimorphisms $(i=1,2)$. Let $N_{1}$ and $N_{2}$ be normal subgroups and $A_{1}$ and $A_{2}$ be subgroups of $G_{1}$ and $G_{2}$, respectively. Then $\sigma: G_{1} \times G_{2} \rightarrow H_{1} \times H_{2}$ is an epimorphism and

$$
\begin{aligned}
& \text { (1) } \sigma\left(\left(\underline{N_{1} \times N_{2}}\right)\left(A_{1} \times A_{2}\right)\right)=\underline{\sigma\left(N_{1} \times N_{2}\right)}\left(\sigma\left(A_{1} \times A_{2}\right)\right) \text {, } \\
& \text { (2) } \sigma\left(\left(\overline{N_{1} \times N_{2}}\right)\left(A_{1} \times A_{2}\right)\right)=\overline{\sigma\left(N_{1} \times N_{2}\right)}\left(\sigma\left(A_{1} \times A_{2}\right)\right) \text {. }
\end{aligned}
$$

Proof. Let $\varphi_{i}: G_{i} \rightarrow H_{i}$ be group epimorphism ( $\left.i=1,2\right)$. Define $\sigma$ by

Then $\sigma$ is an epimorphism.

$$
\sigma: G_{1} \times G_{2} \rightarrow H_{1} \times H_{2}, \quad\left(g_{1}, g_{2}\right) \mapsto\left(\varphi_{1}\left(g_{1}\right), \varphi_{2}\left(g_{2}\right)\right) .
$$

(1) Since $\sigma: G_{1} \times G_{2} \rightarrow H_{1} \times H_{2}$ is an epimorphism, $N_{1}$ and $N_{2}$ are normal subgroups and $A_{1}$ and $A_{2}$ are subgroups of $G_{1}$ and $G_{2}$, respectively, we obtain $\varphi_{1}\left(N_{1}\right)$ and $\varphi_{2}\left(N_{2}\right)$ are normal subgroups and $\varphi_{1}\left(A_{1}\right)$ and $\varphi_{2}\left(A_{2}\right)$ are subgroups of $H_{1}$ and $H_{2}$, respectively. Thus $\varphi_{1}\left(N_{1}\right) \times \varphi_{2}\left(N_{2}\right)$ is a normal subgroup and $\varphi_{1}\left(A_{1}\right) \times \varphi_{2}\left(A_{2}\right)$ is a subgroup of $H_{1} \times H_{2}$, respectively. Then

$$
\begin{aligned}
& \sigma\left(\left(\underline{N_{1} \times N_{2}}\right)\left(A_{1} \times A_{2}\right)\right)=\sigma\left(\left(\underline{N_{1}}\left(A_{1}\right) \times \underline{N_{2}}\left(A_{2}\right)\right) \text {, by Proposition } 12 \text { on } G_{1} \times G_{2},\right. \\
& =\varphi_{1}\left(\bar{N}_{1}\left(A_{1}\right)\right) \times \varphi_{2}\left(N_{2}\left(A_{2}\right)\right) \\
& =\varphi_{1}\left(\overline{N_{1}}\right)\left(\varphi_{1}\left(A_{1}\right)\right) \times \overline{\varphi_{2}}\left(N_{2}\right)\left(\varphi_{2}\left(A_{2}\right)\right), \quad \text { by Theorem } 5 \text { (1), } \\
& \left.\left.=\overline{\left(\varphi_{1}\left(N_{1}\right)\right.} \times \varphi_{2}\left(N_{2}\right)\right) \overline{\left(\varphi_{1}\left(A_{1}\right)\right.} \times \varphi_{2}\left(A_{2}\right)\right) \text {, by Proposition } 12 \text { on } H_{1} \times H_{2} \text {, } \\
& =\underline{\sigma\left(N_{1} \times N_{2}\right)}\left(\sigma\left(A_{1} \times A_{2}\right)\right) \text {. }
\end{aligned}
$$

(2) Similar to the proof of (1).

Proposition 23 Let $\varphi_{i}: G_{i} \rightarrow H_{i}$ be group epimorphisms $(i=1,2)$. Let $N_{1}{ }^{\prime}$ and $N_{2}{ }^{\prime}$ be normal subgroups and $A_{1}{ }^{\prime}$ and $A_{2}{ }^{\prime}$ be subgroups of $H_{1}$ and $H_{2}$, respectively. Then $\sigma: G_{1} \times G_{2} \rightarrow H_{1} \times H_{2}$ is an epimorphism and

$$
\begin{aligned}
& \text { (1) } \left.\sigma^{-1}\left(\underline{\left(N_{1}{ }^{\prime} \times N_{2}{ }^{\prime}\right.}\right)\left(A_{1}{ }^{\prime} \times A_{2}{ }^{\prime}\right)\right)=\underline{\sigma^{-1}\left(N_{1}{ }^{\prime} \times N_{2}{ }^{\prime}\right)}\left(\sigma^{-1}\left(A_{1}{ }^{\prime} \times A_{2}{ }^{\prime}\right)\right) \\
& \text { (2) } \sigma^{-1}\left(\left(\overline{N_{1}{ }^{\prime} \times N_{2}{ }^{\prime}}\right)\left(A_{1}{ }^{\prime} \times{A_{2}}^{\prime}\right)\right)=\overline{\sigma^{-1}\left(N_{1}{ }^{\prime} \times N_{2}{ }^{\prime}\right)}\left(\sigma^{-1}\left(A_{1}{ }^{\prime} \times A_{2}{ }^{\prime}\right)\right)
\end{aligned}
$$

Proof. Similar to the proof of Proposition 22.

Proposition 24 Let $\varphi_{i}: G_{i} \rightarrow H_{i}$ be group epimorphisms ( $i=1,2$ ). Let $N_{i}$, $A_{i}$ be normal subgroups of $G_{i}(i=1,2)$. Then

(1) If $\operatorname{ker} \varphi_{i} \subseteq N_{i} \subseteq A_{i}$, then 


$$
G_{1} / \underline{N_{1}}\left(A_{1}\right) \times G_{2} / \underline{N_{2}}\left(A_{2}\right) \cong H_{1} / \underline{\varphi_{1}\left(N_{1}\right)}\left(\varphi_{1}\left(A_{1}\right)\right) \times H_{2} / \underline{\varphi_{2}\left(N_{2}\right)}\left(\varphi_{2}\left(A_{2}\right)\right),
$$

(2)If $\operatorname{ker} \varphi_{i} \subseteq N_{i}$, then

$$
G_{1} / \overline{N_{1}}\left(A_{1}\right) \times G_{2} / \overline{N_{2}}\left(A_{2}\right) \cong H_{1} / \overline{\varphi_{1}\left(N_{1}\right)}\left(\varphi_{1}\left(A_{1}\right)\right) \times H_{2} / \overline{\varphi_{2}\left(N_{2}\right)}\left(\varphi_{2}\left(A_{2}\right)\right) .
$$

Proof. (1) By the Theorem 7 (1) we get

Hence

$$
G_{1} / \underline{N_{1}}\left(A_{1}\right) \cong H_{1} / \underline{\varphi_{1}\left(N_{1}\right)}\left(\varphi_{1}\left(A_{1}\right)\right) \text { and } G_{2} / \underline{N_{2}}\left(A_{2}\right) \cong H_{2} / \underline{\varphi_{2}\left(N_{2}\right)}\left(\varphi_{2}\left(A_{2}\right)\right) .
$$

$$
G_{1} / \underline{N_{1}}\left(A_{1}\right) \times G_{2} / \underline{N_{2}}\left(A_{2}\right) \cong H_{1} / \underline{\varphi_{1}\left(N_{1}\right)}\left(\varphi_{1}\left(A_{1}\right)\right) \times H_{2} / \underline{\varphi_{2}\left(N_{2}\right)}\left(\varphi_{2}\left(A_{2}\right)\right) .
$$

(2) By the Theorem 7 (2) we get

Hence

$$
G_{1} / \overline{N_{1}}\left(A_{1}\right) \cong H_{1} / \overline{\varphi_{1}\left(N_{1}\right)}\left(\varphi_{1}\left(A_{1}\right)\right) \text { and } G_{2} / \overline{N_{2}}\left(A_{2}\right) \cong H_{2} / \overline{\varphi_{2}\left(N_{2}\right)}\left(\varphi_{2}\left(A_{2}\right)\right) .
$$

$$
G_{1} / \overline{N_{1}}\left(A_{1}\right) \times G_{2} / \overline{N_{2}}\left(A_{2}\right) \cong H_{1} / \overline{\varphi_{1}\left(N_{1}\right)}\left(\varphi_{1}\left(A_{1}\right)\right) \times H_{2} / \overline{\varphi_{2}\left(N_{2}\right)}\left(\varphi_{2}\left(A_{2}\right)\right) .
$$

\section{CONCLUSION}

In this study, we showed that it was possible to apply the rough sets theory to the area of direct products of groups. Then, the concepts of direct product of rough approximations and subgroups in a group were first defined and some of their basic properties were proven. We also showed that for two sets the rough approximations of their direct product were equal to the direct product of their rough approximations. Furthermore, we discussed the structure of direct product of rough approximations of subgroups under homomorphisms between two groups.

\section{REFERENCES}

[1] Pawlak Z. Rough sets, Int J Comput Inform Sci 1982; 11: 341-356.

[2] Pawlak Z. Rough sets and intelligent data analysis, Inform Sci 2002; 147(1-4): 1-12.

[3] Pawlak Z, Skowron A. Rough sets and Boolean reasoning. Inform Sci 2007; 177(1): 41-73.

[4] Zhan J, Liu Q, Herawan T. A novel soft rough set: Soft rough hemirings and corresponding multicriteria group decision making, Applied Soft Computing 2017; 54: 393-402.

[5] Bonikowski Z. Algebraic structures of rough sets, in: W.P. Ziarko (Ed.), Rough Sets, Fuzzy Sets and Knowledge Discovery, Springer-Verlag, Berlin, 1995, pp. 242-247.

[6] Pomykala J, Pomykala J. A. The stone algebra of rough sets, Bull Polish Acad Sci Math 1998 ; 36: 495-508.

[7] Iwinski T. Algebraic approach to rough sets, Bull Polish Acad Sci Math 1987; 35: 673-683.

[8] Kuroki N, Wang PP. The lower and upper approximations in a fuzzy group, Inform Sci 1996; 90 : 203-220.

[9] Bağırmaz N, Özcan AF, İçen İ. Rough Approximations in a Topological Group, Gen Math Notes 2016; 36(2): 1-18. 
[10] Cheng W, Mo Z, Wang J. Notes on "the lower and upper approximations in a fuzzy group" and "rough ideals in semigroups", Inform Sci 2007; 177: 5134-5140.

[11] Wang C, Chen D. A short note on some properties of rough groups, Comput Math Appl 2010; 59: 431-436.

[12] Wang C, Chen D, Hu Q. On rough approximations of groups, Int J Mach Learn \& Cyber, 2013; 4(5): 445-449.

[13] Wang Z, Shu L. The lower and upper approximations in a grop, International Journal of Mathematical and Computational Sciences 2012; 6: 158-162.

[14] Li F, Zhang Z. The Homomorphisms and Operations of Rough Groups, The Scientific World Journal, Volume 2014, Article ID 507972, 6 pages.

[15] Biswas R, Nanda S. Rough groups and rough subgroups, Bull Polish Acad Sci Math 1994; 42: 251-254.

[16] Miao D, Han S, Li D, and Sun L. Rough Group, Rough Subgroup and Their Properties, D. Ślkezak et al. (Eds.): RSFDGrC 2005, LNAI 3641, pp. 104--113, 2005.C Springer-Verlag Berlin Heidelberg, 2005.

[17] Bağırmaz N, Özcan AF. Rough semigroups on approximation spaces, International Journal of Algebra 2015; 9(7): 339-350.

[19] Feyzioğlu AK. A course on algebra, Boğaziçi University Publication, İstanbul, 1990. 